\title{
Graph theoretic analysis of protein interaction networks of eukaryotes
}

\author{
K.-I. Goh*, B. Kahng*,† and D. Kim* \\ * School of Physics and ${ }^{\dagger}$ Program in Bioinformatics, \\ Seoul National University, Seoul 151-747, Korea
}

(Dated: June 15, 2004)

\begin{abstract}
Thanks to recent progress in high-throughput experimental techniques, the datasets of large-scale protein interactions of prototypical multicellular species, the nematode worm Caenorhabditis elegans and the fruit fly Drosophila melanogaster, have been assayed. The datasets are obtained mainly by using the yeast hybrid method, which contains false-positive and false-negative simultaneously. Accordingly, while it is desirable to test such datasets through further wet experiments, here we invoke recent developed network theory to test such high throughput datasets in a simple way. Based on the fact that the key biological processes indispensable to maintaining life are universal across eukaryotic species, and the comparison of structural properties of the protein interaction networks (PINs) of the two species with those of the yeast PIN, we find that while the worm and the yeast PIN datasets exhibit similar structural properties, the current fly dataset, though most comprehensively screened ever, does not reflect generic structural properties correctly as it is. The modularity is suppressed and the connectivity correlation is lacking. Addition of interlogs to the current fly dataset increases the modularity and enhances the occurrence of triangular motifs as well. The connectivity correlation function of the fly, however, remains distinct under such interlogs addition, for which we present a possible scenario through an in silico modeling.
\end{abstract}

\section{Introduction}

In the last few years graph theoretic methods to understand complex biomolecular systems have been developed very rapidly [4]. Such a development has made advances toward uncovering the organizing principles of cellular networks in post-genomic biology. The cellular components such as genes, proteins, and other biological molecules, connected by all physiologically relevant interactions, form a full weblike molecular architecture in a cell. In such an architecture, genes play a central role, which are expressed through proteins. Proteins rarely act alone, rather they cooperate with others to act physiologically. Thus protein interactions play pivotal roles in various aspects of the structural and functional organizations and their complete description would be the first step toward a thorough understanding of the web of life. Proteins are viewed as nodes of a complex protein interaction network (PIN) in which two proteins are linked if they physically contact with each other. The graph theoretic approach has been useful to understand intricate interwoven structures of the PIN [9, 11, 23]. The key biological processes indispensable to maintaining life are universal across eukaryotic species since many involved genes are evolutionarily conserved [1]. Using this property, one can test a newly discovered dataset if it really contains more or less complete information of protein interactions. Moreover, this in silico approach offers one the candidates of protein interaction pairs, of which the number is considerably reduced compared with the total combinatorial pairs. Thus, the graphic theoretic analysis would provide a useful guide for further wet studies of protein interactions.

Species with sequenced genome such as the yeast Saccharomyces cerevisiae provide important test beds for the study of the PIN. Thanks to recent progress in the high-throughput experimental techniques such as the yeast two-hybrid assay 8, 21] and the mass spectroscopy 5, 7], the dataset of the yeast PIN has been firmly established [13, 17]. Very recently, large-scale protein interactions of multicellular species, the nematode worm Caenorhabditis elegans [10] and the fruit fly Drosophila melanogaster [ 6 ], have been assayed. While those datasets, mainly based on the yeast two-hybrid assay, need physiological proof, they contain large-scale proteins and protein interactions, making graph theoretic study possible. In this paper, we analyze those datasets and compare them with the more-established set of interactions in the budding yeast [17]. Our graph theoretic analysis suggests that the present interaction dataset of the fruit fly, based on the yeast two-hybrid ( $\mathrm{Y} 2 \mathrm{H})$ assay, may have left out a significant part of protein interactions, though most comprehensively screened ever. Such conclusion has been reached by the comparison of the generic features of the PIN, the modularity and the connectivity correlations, across the three species. For the fly, those quantities behave distinctively: The modularity is suppressed and the connectivity correlation is lacking. Such distinct behavior can be overcome partially by the addition of yeast interlogs into the fly dataset.

\section{Materials and Methods}

Graph theory terminology. (i) Network is composed of vertices and edges. In the protein interaction network, vertices represent proteins and edges protein interactions. (ii) Degree is the number of edges connected to a given vertex. The degree distribution $p_{d}(k)$ is the fraction of vertices having $k$ degrees. (iii) Clustering coefficient of a node is defined as $C_{i}=2 e_{i} / k_{i}\left(k_{i}-1\right)$, where $e_{i}$ is the number of connections among the $k_{i}$ neighbors of a vertex $i$. Clustering function $C(k)$ is the mean value of $C_{i}$ over the vertices with degree $k$, while the clustering coefficient 

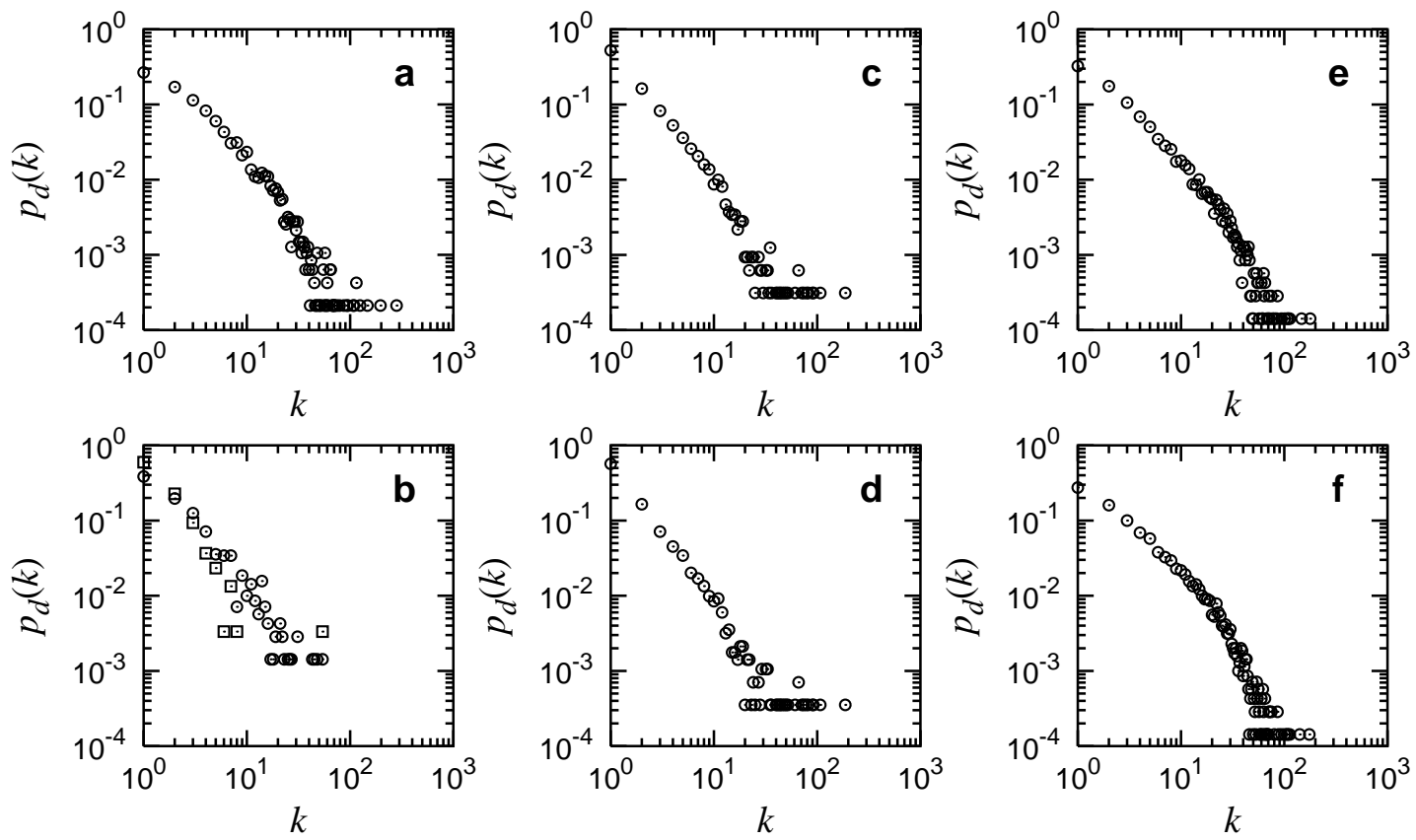

Fig. 1: The degree distributions $p_{d}(k)$ for (a) the yeast, (b) the prokaryotes Helicobacter pylori (o) and Escherichia coli ( $\square$ ), (c) the worm (Worm-All), (d) the Y2H subset of the worm dataset (Worm-Y2H), (e) the fly, and (f) the Fly+Interlog dataset.

$C$ is the mean of $C_{i}$ over all vertices. When the network contains hierarchical and modular structures within it, it is known that the clustering function $C(k)$ behaves as $C(k) \sim k^{-\beta}$ for large $k$ [16]. (iv) $\left\langle k_{\mathrm{nn}}\right\rangle(k)$ is the mean degree of the neighbors of a vertex with degree $k$. It is known that $\left\langle k_{\mathrm{nn}}\right\rangle(k) \sim k^{-\nu}$ with $\nu>0$ for the Internet and the protein interaction network [11, 15], implying that vertices with large degree tend to connect to the ones with small degree. Such a network is called dissortative network. Besides this quantity, the ep $\mathrm{x} r$ has been introduced [14] to characterize the degree-degree correlation between the two vertices located at the ends of an edge, which is defined as

$$
r=\frac{\left\langle k_{1} k_{2}\right\rangle-\left\langle\left(k_{1}+k_{2}\right) / 2\right\rangle^{2}}{\left\langle\left(k_{1}^{2}+k_{2}^{2}\right) / 2\right\rangle-\left\langle\left(k_{1}+k_{2}\right) / 2\right\rangle^{2}}
$$

where $k_{1}$ and $k_{2}$ are the degrees of two vertices at the ends of an edge, and $\langle\cdots\rangle$ denotes the average over all edges.

The protein interaction network datasets. We used the yeast subset of the interaction data compiled in the Database of Interacting Proteins (DIP) as of January 2004 (http://dip.doe-mbi.ucla.edu) 17. The datasets for the worm and the fly are obtained from the works of $\mathrm{Li}$ et al. 10] and Giot et al. 6], respectively. For the worm, we consider two different versions, the one consisting of only the interactions from the $\mathrm{Y} 2 \mathrm{H}$ screens (referred to as Worm-Y2H network in this paper) and the other the full network supplied by $\mathrm{Li}$ et al. 10. (referred to as Worm-All network). The characteristics of each dataset and the values of the graphic theoretic quantities are tabulated in Table 1

Orthologous gene assignment. For cross-species ortholog information, we used the information from the KOG database 19], a eukaryotic extension of the Clusters of Orthologous Genes (COG) database (http://www.ncbi.nlm.nih.gov/COG/new/).

Yeast interlogs in fly. Having identified the yeast-fly orthologs, we look for the interactions in the yeast network between those yeast proteins both having orthologs in the fly network. Such orthologous interactions are called the interlogs. If the corresponding

Table 1: Protein interaction network datasets. Tabulated are for each dataset the size of proteome $N_{\text {proteome }}$, the number of proteins $N$ and the number of protein-protein interactions $L$ in the dataset, the mean degree $\langle k\rangle$, the clustering coefficient $C$, the assortativity $r$, and the number of proteins forming the largest cluster $N_{1}$. The self-interactions are excluded throughout.

\begin{tabular}{ccccc}
\hline \hline & Yeast & Worm-Y2H & Worm-All & Fly \\
\hline$N_{\text {proteome }}$ & 6195 & 22246 & 22246 & 16206 \\
$N$ & 4714 & 2835 & 3216 & 7055 \\
$L$ & 14857 & 4438 & 50444 & 20947 \\
$\langle k\rangle$ & 6.3 & 3.1 & 3.4 & 5.9 \\
$C$ & 0.12 & 0.047 & 0.15 & 0.014 \\
$r$ & -0.14 & -0.16 & -0.13 & -0.036 \\
$N_{1}$ & 4627 & 2601 & 2898 & 6929 \\
\hline \hline
\end{tabular}



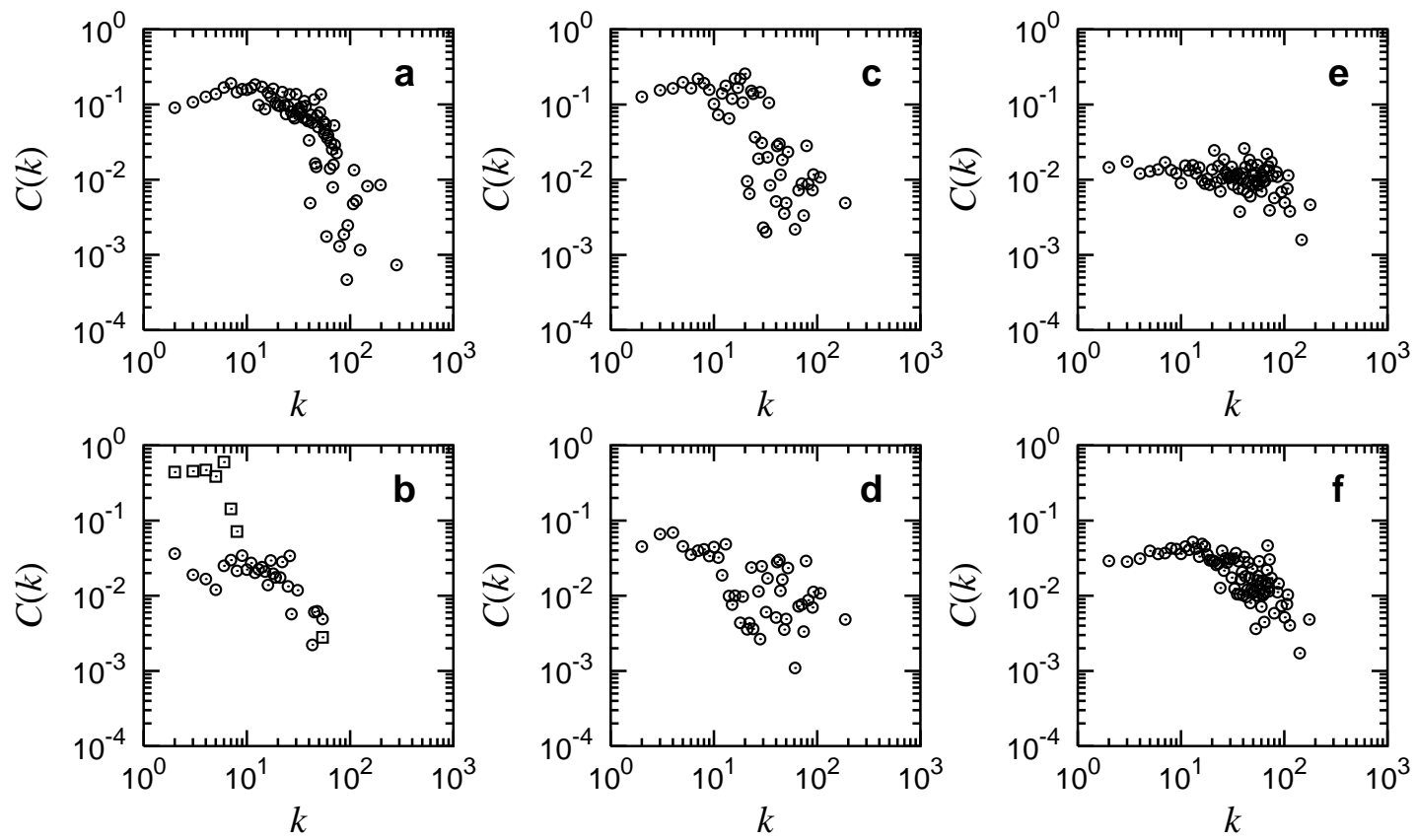

Fig. 2: The local clustering function $C(k)$ for (a) the yeast, (b) the bacteria H. pylori (o) and E. coli ( $\square)$, (c) the worm (Worm-All), (d) the Worm-Y2H dataset, (e) the fly, and (f) the Fly+Interlog dataset. The abscissae and ordinates are fixed for clear comparison.

fly interaction is present, we call it an overlap interlog. If not, we call it a potential interlog. Note that the ortholog relationship is not always one-to-one, resulting in multiple interlogs for a given yeast interaction. For in silico analysis on the effect of the addition of potential interlogs in the fly network, we include on average one potential interlog per yeast interaction. Specifically, for each yeast interaction A-B having no overlap interlog, each potential interlog is added in the fly network with probability $1 /\left(o_{A} o_{B}\right)$, where $o_{X}$ is the number of fly ortholog(s) of the yeast gene X. The network obtained in this way is referred to as Fly+Interlog network hereafter. The full list of the 408 overlap and the 55176 potential interlogs are available on the web (http://komplex0.snu.ac.kr/pin/yeast-fly-interlog.xls).

\section{Results}

Degree distributions. In Fig. 1 we plot the degree distributions of diverse protein interaction networks, all of which display the scale-free behavior, fitting well to the generalized Pareto formula, $p_{d}(k) \sim\left(k+k_{0}\right)^{-\gamma}$, almost indistinguishable with each other. While the degree distribution is a fundamental quantity in graph theory, it deals with global network structure, so it does not give detailed information on structural property.

\section{Modularity}

A cellular function is achieved by a set of related proteins, usually forming a pathway or a complex. Such functional module manifests itself as a localized dense subgraph within the whole cellular network. The presence of modules and their hierarchical organization can be visualized by the local clustering function $C(k)$ 16]. For the yeast PIN, $C(k)$ exhibits a plateau for small $k$ and falls off rapidly for large $k$, reflecting the modular structure bridged by the hubs (Fig. 2a). The similar pattern is observed in the worm (Fig. 2c) and the two prokaryotic species, H. pylori and E. coli (Fig. 2b). Note that the worm dataset contains the yeast interlogs. For the fly $\mathrm{Y} 2 \mathrm{H}$ data, however, $C(k)$ behaves distinctively, almost constant for all $k$ (Fig. 2e). To understand this discrepancy, we add the potential yeast interlogs into the current fly $\mathrm{Y} 2 \mathrm{H}$ dataset. Then $C(k)$ behaves in a similar fashion to other dataset, showing a moderate plateau for small $k$ and rapid decrease for large $k$, albeit the altitude of the plateau, which is roughly the clustering coefficient $C$, is not as high as in the yeast and the worm (Fig. 2f). To find the role of the interlogs in the worm, we consider the Worm-Y2H dataset, and plot its $C(k)$ in Fig. 2d. Indeed, the signature character of $C(k)$ is lost, in particular, the plateau for small $k$ almost disappears, implying the yeast-interlogs play a role of forming modules, where proteins are closely linked each other.

Conservation rate of interactions. We count how many yeast interactions are actually conserved in orthologous form in both the worm and the fly. The conservation rate found in this way for the $\mathrm{Y} 2 \mathrm{H}$ screen dataset is surprisingly low; $2.7 \%$ for the worm (Worm$\mathrm{Y} 2 \mathrm{H}$ ) and $3.8 \%$ for the fly. For the worm, we note that such low coverage is in part due to the insufficient num- 

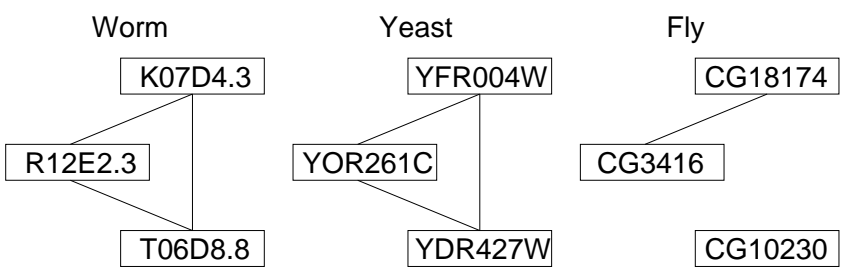

Fig. 3: Conservation of interaction motif. Shown in the middle is a triangular interaction subgraph within the yeast involving in ubiquitin-dependent protein catabolism. Corresponding orthologous counterpart in the worm and the fly are also shown. This motif is conserved in the worm $\mathrm{Y} 2 \mathrm{H}$ data, while only a single interaction is detected in the fly data.

ber of baits used in the experiment (3,024 baits, 833 out of which are present in the network). When we consider the conservation of triangular interaction patterns, a basic unit of cooperative functional module 12], only 3 out of 1731 are conserved in the worm, while none in the fly (Fig. 3). The lack of conserved interaction motifs in the fly data suggests that the current fly network misses some of important cooperative aspects of the cellular network in the fly. The effort to fill this gap is timely.

Motif structure. Since the modularity manifested by $C(k)$ is closely related to the formation of triangles in the network, here we further perform network motif analysis for the three species datasets. The network motifs are small recurring subgraphs which are overrepresented in a given network and are believed to provide the basic evolutionary and functional signatures of the network [12. Since it was recently discovered that the motif constituents are more conserved during evolution than the rest 24], one would expect the density of each motif to be close to each other across the three species. From the comparison of the columns for Yeast, Worm-All, and Fly in Table 2 we can see that the triangle motif is relatively not abundant in Fly, while the square motif is. Thus, the absolute magnitude of the clustering function is smaller for the fly than for the yeast or the worm. The density of the triangle motif is higher in the Fly+Interlog dataset, indicating that the clustering coefficient is enhanced overall by the addition of the interlogs of the fly.

In Table 2 we have summarized the motif structure for each network. We follow Milo et al. 12] to calculate the two scores, $Z$ - and $E$-score, defined as $Z=(N-$ $\left.N_{\text {random }}\right) / \sigma_{\text {random }}$ and $E=\left(N-N_{\text {random }}\right) / N_{\text {random }}$, respectively, and use the following two criteria to specify whether a subgraph is a motif or an anti-motif (an antimotif is a subgraph significantly underrepresented in the network):

(i) The probability that $N$ is observed in randomized network is smaller than 0.01 .

(ii) $|E|>E_{0}$, where we set the threshold $E_{0}=0.5$, rather than $E_{0}=0.1$ in Milo et al. 12].

Here, $N_{\text {random }}$ and $\sigma_{\text {random }}$ are the expected number of occurrence in the randomized version of the network and their standard deviation obtained from 1000 samples respectively, where the randomization is performed by the switching method [12]. In calculating them for the 4-node subgraphs, the numbers of 3-node subgraphs are fixed to be those of the original networks. For the Fly+Interlog network, 10 realizations of interlog addition (see Method) are averaged.

\section{Degree-degree correlation}

The mean neighbor degree function $\left\langle k_{\mathrm{nn}}\right\rangle(k)$ is useful in understanding the degree-degree correlation in a network. In Fig. 4 we plot $\left\langle k_{\mathrm{nn}}\right\rangle(k)$ for each dataset. For the yeast, it is known that $\left\langle k_{\mathrm{nn}}\right\rangle(k)$ decreases with increasing $k$ 11], which turns out to be also true for some prokaryotic species, too (Figs. 4a-b). Such a behavior in $\left\langle k_{\mathrm{nn}}\right\rangle(k)$ is also observed for the worm (Figs. 4c-d), however, it is flat for the fly, implying lack of correlation (Fig. 4e). Such distinct behavior for the fly is robust under the addition of the interlogs (Fig. 4f), which suggests the lack of correlation in the fly network could be intrinsic, even though we cannot exclude the possibility that it is again the artifact of the incompleteness of the data. The hypothesis that the lack of correlation could be intrinsic may be supported by the following observations.

Effect of diversification of gene function on $\left\langle k_{\mathrm{nn}}\right\rangle(k)$. While the pattern of $C(k)$ of the fly becomes similar to those of the yeast and the worm by the addition of the interlogs, that of $\left\langle k_{\mathrm{nn}}\right\rangle(k)$ remains distinct. Thus here we investigate if such a flat behavior is intrinsic through an in silico model, finding that indeed, the decreasing behavior of $\left\langle k_{\mathrm{nn}}\right\rangle(k)$ becomes moderated through the network evolution with the duplication and divergence processes. Homologs in a genome are thought to result from the gene duplication event, which is usually followed by the diversification to lower the redundancy. Some computer models aiming to mimic these processes in proteome evolution exist in the literature 18, 22]. We investigate how the diversification process affects the topological property of the proteome network, in particular, the degree-degree correlation in terms of $\left\langle k_{\mathrm{nn}}\right\rangle(k)$. To this end, we perform following procedures motivated by Vázquez et al. 22]:

1. Starting with the yeast protein network, at each step, a protein $\mathrm{A}$ is chosen randomly and is duplicated as $\mathrm{A}^{\prime}$. Then the protein $\mathrm{A}$ and $\mathrm{A}^{\prime}$ share common neighbors.

2. For each neighboring protein of $A$ and $A^{\prime}$, one of edges connected to either $\mathrm{A}$ or $\mathrm{A}^{\prime}$ is removed with equal probability.

3. Repeat 1-2 until the number of proteins reaches $\sim 20,000$, the approximate sizes of the worm and the fly proteome.

Note that in this procedure, the number of proteins increases while the number of interactions stays still. Thus 
Table 2: Network motif structure of the three species. Tabulated is the number of each subgraph present in the network. According to its $Z$ - and $E$-score, the significant motifs (M) and anti-motifs (AM) are indicated.

\begin{tabular}{|c|c|c|c|c|c|}
\hline & Yeast & Worm-Y2H & Worm-All & Fly & Fly+Interlog \\
\hline$\Omega$ & 329961 & 81205 & 87294 & 413926 & $520704 \pm 1358$ \\
\hline \multirow{2}{*}{$\Delta$} & 7136 & \multirow[t]{2}{*}{366} & 1512 & \multirow[t]{2}{*}{1549} & $3504 \pm 40$ \\
\hline & $\mathrm{M}(\mathrm{Z}=80, \mathrm{E}=3.3)$ & & $\mathrm{M}(\mathrm{Z}=29, \mathrm{E}=2.5)$ & & $\mathrm{M}(\mathrm{Z}=45, \mathrm{E}=1.4)$ \\
\hline & 4081023 & 604723 & 680485 & 7378808 & $971960 \pm 37157$ \\
\hline$\widehat{D}$ & 9024723 & 2129609 & 2157048 & 6315922 & $7409320 \pm 24476$ \\
\hline \multirow{2}{*}{ 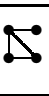 } & 368730 & 46050 & 58520 & \multirow[t]{2}{*}{160846} & \multirow[t]{2}{*}{$263324 \pm 2617$} \\
\hline & $\mathrm{AM}(\mathrm{Z}=-122, \mathrm{E}=-0.7)$ & & $\mathrm{AM}(\mathrm{Z}=-59, \mathrm{E}=-0.7)$ & & \\
\hline \multirow{2}{*}{ 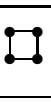 } & 21806 & 4350 & 4686 & \multirow{2}{*}{$\begin{array}{c}54100 \\
M(Z=81, E=2.1)\end{array}$} & $60648 \pm 206$ \\
\hline & & $\mathrm{M}(\mathrm{Z}=9.5, \mathrm{E}=0.6)$ & & & $\mathrm{M}(\mathrm{Z}=60, \mathrm{E}=1.3)$ \\
\hline \multirow{2}{*}{ 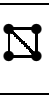 } & 27455 & \multirow[t]{2}{*}{1505} & 4120 & 4029 & \multirow[t]{2}{*}{$9313 \pm 228$} \\
\hline & $\mathrm{AM}(\mathrm{Z}=-49, \mathrm{E}=-0.7)$ & & $\mathrm{AM}(\mathrm{Z}=-25, \mathrm{E}=-0.6)$ & $\mathrm{M}(\mathrm{Z}=12, \mathrm{~N}=0.8)$ & \\
\hline \multirow{2}{*}{ 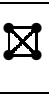 } & 5259 & \multirow[t]{2}{*}{30} & 1563 & 82 & $914 \pm 35$ \\
\hline & & & $\mathrm{M}(\mathrm{Z}=10, \mathrm{E}=0.8)$ & $\mathrm{M}(\mathrm{Z}=11, \mathrm{E}=3.5)$ & $\mathrm{M}(\mathrm{Z}=40, \mathrm{E}=6.0)$ \\
\hline
\end{tabular}
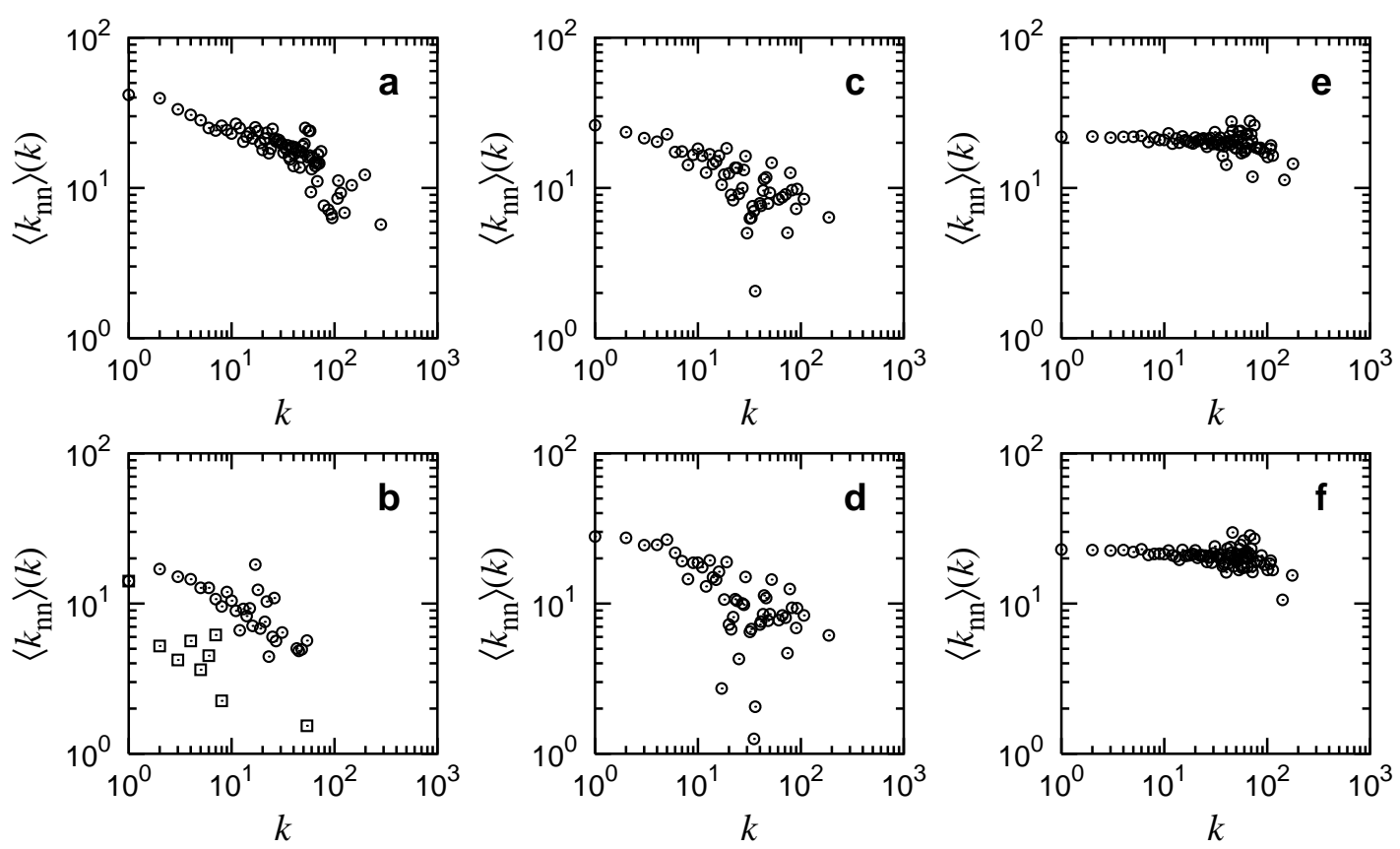

Fig. 4: The average neighbor degree function $\left\langle k_{\mathrm{nn}}\right\rangle(k)$ for (a) the yeast, (b) the prokaryotes H. pylori (o) and E. coli ( $\square$ ), (c) the worm (Worm-All), (d) the Y2H subset of the worm (Worm-Y2H), (e) the fly, and (f) the Fly+Interlog dataset. The abscissae and ordinates are fixed for clear comparison.

the average degree decreases as the size of proteome increases. Such decrease will be compensated by, e.g., the acquisition of new interactions between existing proteins via mutation. However, we do not take such a process into account, to single out the effect of the diversification only.

The result of simulation is shown in Fig. 5 The local clustering function $C(k)$ is simply shifted downward, due to the overall decrease of the edge density. On the other hand, the average neighbor degree $\left\langle k_{\mathrm{nn}}\right\rangle(k)$ decreases as $k$ but with a smaller rate, indicating that the diversification process can, although not perfectly, neutralize the connectivity correlation. Furthermore, if we assume that the establishment of new interactions follows the preferential attachment [3] or random attachment, the overall correlation would diminish eventually. 

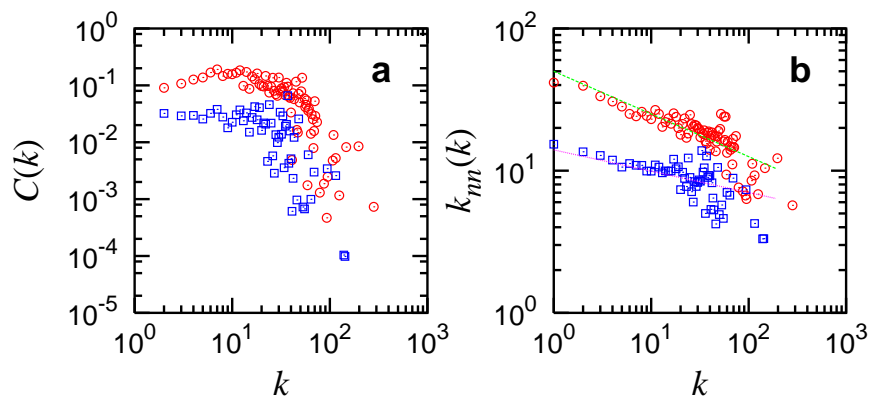

Fig. 5: Effect of gene function diversification in (a) $C(k)$ and (b) $\left\langle k_{\mathrm{nn}}\right\rangle(k)$. Red circles are the data of the original yeast network and the blue squares those after running the diversification procedures in silico. The slope of the straight line (the rate of decrease) in (b) is -0.3 (top, green) and -0.15 (bottom, magenta), respectively.

Effect of bait selection on $\left\langle k_{\mathrm{nn}}\right\rangle(k)$. There has been an argument that the apparent decreasing trend in $\left\langle k_{\mathrm{nn}}\right\rangle(k)$ is an artifact from the limited selection of baits in the two-hybrid experiment 2]. Indeed, $\mathrm{Li}$ et al. 10] had selected the baits with their own criteria, mainly based on the biological indispensability and the potential applicability to the human therapeutics. To check this hypothesis in silico, we sampled the $30 \%$ subset of 4950 baits identified in Giot et al.'s fly network [ 6 ] and reconstructed the network only with the interactions associated with the sampled baits. We sampled in two different ways; the random sampling and the biased sampling toward the highly connected baits (the sampling probability is proportional to the number of bait-interactions). Both data sets generate the decreasing trend in $\left\langle k_{\mathrm{nn}}\right\rangle(k)$ (Fig. 6). One can see that even though the original network has the null slope in $\left\langle k_{\mathrm{nn}}\right\rangle(k)$, the negative slope develops in the sampled ones, demonstrating that the insufficient use of the bait can produce artifactual correlation in the connectivity. If this scenario holds, one conjecture that $\left\langle k_{\mathrm{nn}}\right\rangle(k)$ curve will become flatter as the interaction data accumulates and becomes more complete.

\section{Summary and discussion}

We have investigated in detail the structural properties of the protein interaction networks of three eukaryotic species, the budding yeast, the nematode worm, and the fruit fly. In particular, we have focused on the comparative assessment of the modularity and the degree-degree correlation for those networks. We found that while the worm dataset behaves similarly to the yeast for the two graph theoretic quantities, the fly does not. The difference might be attributed to the presence (absence) of the yeast-interlogs in the current worm (fly) dataset. For the fly dataset, the modularity is suppressed and the connectivity correlation is lacking. We found that the clustering function can be restored to those of the yeast dataset by the addition of interlogs selected randomly among the candidates to the current dataset. We also performed motif analysis for the three species, finding that the density of the triangle motif is increased by the addition of the interlogs to the current fly dataset. Finally, the candidates of the protein interactions of the fly are provided in the supplementary materials, which could be useful in finding protein interactions missed in the current fly dataset.

This work is supported by the KOSEF grant No. R142002-059-01000-0 in the ABRL program and the MOST grant No. M1 03B500000110.
[1] Alberts, B., Bray, D., Johnson, A., Lewis, J., Raff, M., Roberts, K. and Walter, P. (1998) Essential Cell Biology Garland, New York.

[2] Aloy, P. and Russell, P. B. (2002) Potential artefacts in protein-interaction networks. FEBS Lett. 530, 253-254.

[3] Barabási, A.-L., and Albert, R. (1999) Emergence of scaling in random networks. Science 286, 509-512.

[4] Barabási, A.-L. and Oltvai, Z. N. (2004) Network biology: understanding the cell's functional organization. Nat. Rev. Genet. 5, 101-114.

[5] Gavin, A.-C., Bosche, M., Krause, R., Grandi, P., Marzioch, M., Bauer, A., Schultz, J., Rick, J. M., Michon, A. M., Cruciat, C. M., et al. (2002) Functional organization of the yeast proteome by systematic analysis of protein complexes. Nature 415, 141-147.

[6] Giot, L., Bader, J. S., Brouwer, C., Chaudhuri, A., Kuang, B., Li, Y., Hao, Y. L., Ooi, C. E., Godwin, B., Vitols, E., et al. (2003) A protein interaction map of Drosophila melanogaster. Science 302 1727-1736.

[7] Ho, Y., Gruhler, A., Heilbut, A., Bader, G. D., Moore, L., Adams, S. L., Millar, A., Taylor, P., Bennett, K.,
Boutilier, K., et al. (2002) Systematic identification of protein complexes in Saccharomyces cerevisiae by mass spectrometry. Nature 415, 180-183.

[8] Ito, T., Chiba, T., Ozawa, R., Yoshida, M., Hattori, M. and Sakaki, Y. (2001) A comprehensive two-hybrid analysis to explore the yeast protein interactome. Proc. Natl. Acad. Sci. U.S.A. 98, 4569-4574.

[9] Jeong, H., Mason, S. P., Barabási, A.-L. and Oltvai, Z. N. (2001) Lethality and centrality in protein networks. Nature 411, 41-42.

[10] Li, S., Armstrong, C. M., Bertin, N., Ge, H., Milstein, S., Boxem, M., Vidalain, P. O., Han, J. D., Chesneau, A., Hao, T., et al. (2004) A map of the interactome network of the metazoan C. elegans. Science 303, 540-543.

[11] Maslov, S. and Sneppen, K. (2002) Specificity and stability in topology of protein networks. Science 296, 910913.

[12] Milo, R., Shen-Orr, S., Itzkovitz, S., Kashtan, N., Chklovskii, D. and Alon, U. (2002) Network motifs: simple building blocks of complex networks. Science 298, 824-827. 

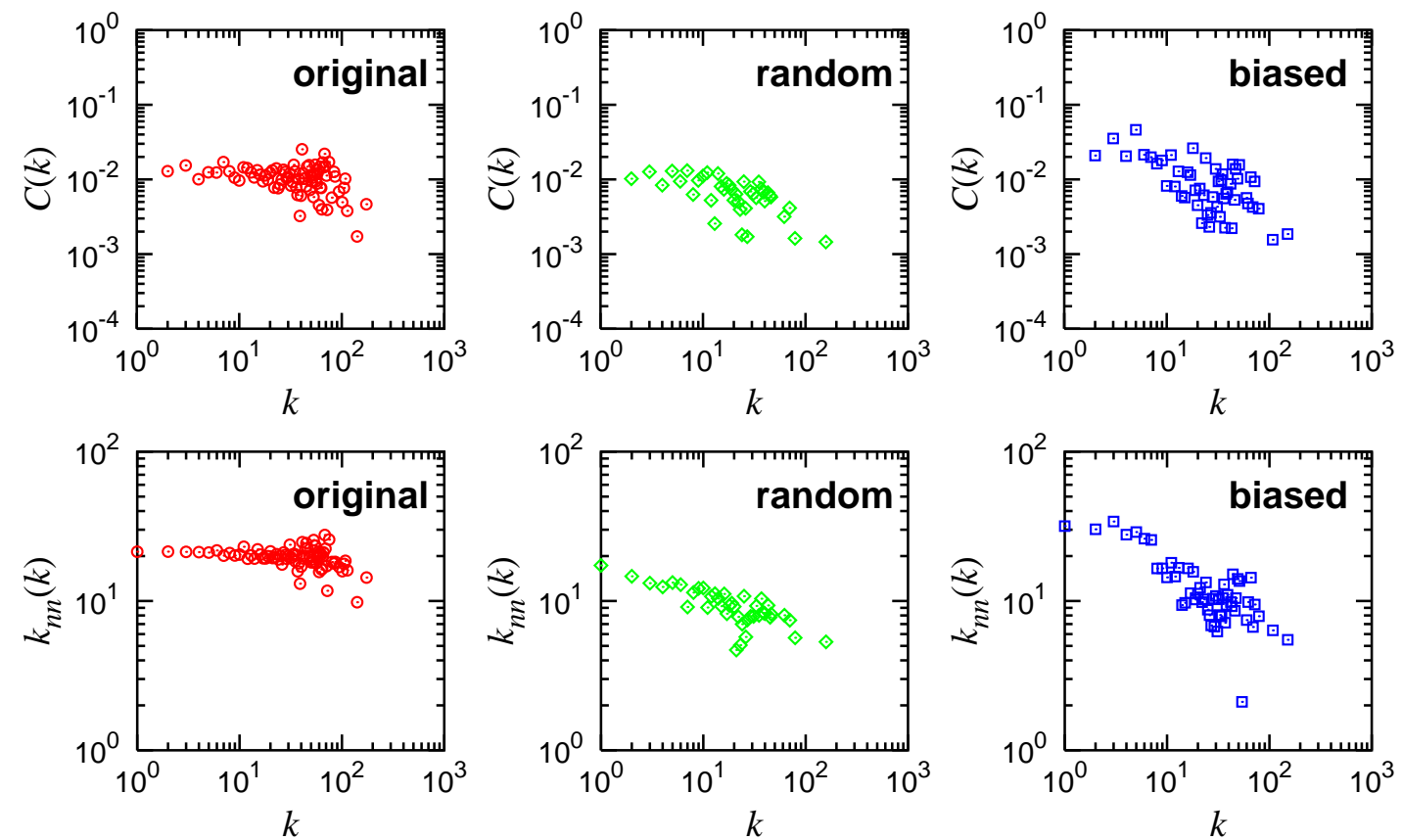

Fig. 6: Effect of bait selection. Red circle is for the full data, green diamond the randomly sampled one, blue square the biased sampled one.

[13] Mewes, H. W., Amid, C., Arnold, R., Frishman, D., Guldener, U., Mannhaupt, G., Munsterkotter, M., Pagel, P., Strack, N, Stumpflen, V., et al., (2004) MIPS: analysis and annotation of proteins from whole genomes. Nucl. Acids Res. 32, D41-D44.

[14] Newman, M. E. J. (2002) Assoratative mixing in networks. Phys. Rev. Lett. 89, 208701.

[15] Pastor-Satorras, R., Vázquez, A. and Vespignani, A. (2001) Dynamical and correlation properities of the Internet, Phys. Rev. Lett. 87, 258701.

[16] Ravasz, E., Somera, A. L., Mongru, D. A., Oltvai, Z. N. and Barabási, A.-L. (2002) Hierarchical organization of modularity in metabolic networks. Science 297, 15511555 .

[17] Salwinski, L., Miller, C. S., Smith, A. J., Pettit, F. K., Bowie, J. U. and Eisenberg, D. (2004) The Database of Interacting Proteins: 2004 update. Nucl. Acids Res. 32, D449-D451.

[18] Solé, R. V., Pastor-Satorras, R., Smith, E. and Kepler, T. (2002) A model of large-scale proteome evolution. Adv. Complex Syst. 5, 43-54.

[19] Tatusov, R. L., Fedorova, N. D., Jackson, J. D., Jacobs, A. R., Kiryutin, B., Koonin, E. V., Krylov, D. M., Mazumder, R., Mekhedov, S. L., Mikolskaya, A. N., et al.
(2003) The COG database: an updated version includes eukaryotes. BMC Bioinformatics 4, 41.

[20] Tong, A. H. Y., Drees, B., Nardelli, G., Bader, G. D., Brannetti, B., Castagnoli, L., Evangelista, M., Ferracuti, S., Nelson, B., Paoluzi, S., et al. (2001) A combined experimental and computational strategy to define protein interaction networks for peptide recognition modules. Science 295, 321-324.

[21] Uetz, P., Giot, L., Cagney, G., Masfield, T. A., Judson, R. S., Knight, J. R., Lockshon, D., Narayan, V., Srinivasan, M, Pochart, P., et al. (2000) A comprehensive analysis of protein-protein interactions in Saccharomyces cerevisiae. Nature 403, 623-627.

[22] Vázquez, A., Flammini, A., Maritan, A. and Vespignani, A. (2003) Modelling of protein interaction networks. Complexus 1, 38-42.

[23] Wagner, A. (2001) The yeast protein interaction network evolves rapidly and contains few redundant duplicate genes. Mol. Biol. Evol. 18, 1283-1292.

[24] Wuchty, S., Oltvai, Z. N. and Barabási, A.-L. (2003) Evolutionary conservation of motif constituents in the yeast protein interaction network. Nat. Genet. 35, 176-179. 